\title{
Quality of Life and Disability in Chinese People Living with Severe Mental Illness
}

\author{
Jiankui Lin $^{1} \cdot$ Yingchun Zeng ${ }^{2}$
}

Received: 13 August 2015/Accepted: 7 October 2015/Published online: 15 October 2015

(C) Springer India Pvt. Ltd. 2015

\begin{abstract}
Severe mental illness (SMI) drastically affects various areas of life. People living with SMI report general impairment in their functioning and poor quality of life. The aim of this study is to describe the prevalence of disability and quality of life in a cohort of Chinese people living with SMI. This was a cross-sectional study. Assessment scales included the World Health Organization (WHO) Disability Assessment Schedule-II, the WHO Quality of Life-BREF version, and the Positive and Negative Syndrome Scale. A total of 158 subjects participated in this study, who were mainly suffering from schizophrenia or related disorders $(n=73,46.2 \%)$. The prevalence of self-reported disability was as high as $69.6 \%$ $(\mathrm{n}=110)$. Subjects perceived higher levels of disability in the aspects of social participation, getting along with people and dealing with life activities. Overall, the total disability scores were significantly correlated with patients' QOL and symptom severity (all $P$ values $<0.001$ ). Older age was a significant risk factor for disability (OR 1.425 , $95 \%$ CI 1.016-1.999). Shorter duration of current hospitalization was a significant protecting factor for disability (OR 0.704, $95 \%$ CI 0.508-0.974). This study found the prevalence of disability among Chinese people living with SMI was high. When the disability was more severe, it was significantly correlated with greater symptom severity and poorer quality of life. Statistically significant predictors of
\end{abstract}

Yingchun Zeng

chloezengyc@hotmail.co.uk

1 Department of Nursing, Guangzhou Psychiatric Hospital, Guangzhou, China

2 Department of Obstetrics, The Third Affiliated Hospital of Guangzhou Medical University, No 63 DuoBao Road, Liwan District, Guangzhou 510150, Guangdong Province, China disability included older age and longer duration of current hospitalization. Future psychiatric rehabilitation intervention studies should be conducted to reduce the impact of disability on quality of life and other health outcomes for people living with SMI.

Keywords Disability - Quality of life - Symptom severity $\cdot$ Severe mental illness

\section{Introduction}

Severe mental illness (SMI) refers to illnesses where psychosis occurs, including schizophrenia, bipolar disorder, schizoaffective disorder, major depressive disorder, and other psychoses [1]. The World Health Organization (WHO) estimates the number of people suffering from SMI ranges from 1 to $3 \%$ [2]. In China, it is estimated that approximately $4 \%$ of adults, or 16 million people experience SMI [3]. It is increasingly recognized that patients with SMI have been found to suffer from impaired QOL [4]. QOL measures are considered useful information for planning and evaluating clinical interventions [5].

Schizophrenia, one type of SMI, has been recognized as the leading cause of disability among the major causes of disability [6]. Common types of disability include disruption in social functioning (i.e. inability to maintain normal social involvement) and cognitive impairment [7, 8]. Various research has indicated that symptom severity of SMI is a significant correlate of quality of life [9, 10]. More severe disability and greater positive symptom severity are correlated with decreased quality of life for people living with SMI $[7,11]$.

Patients with SMI report general impairment in their functioning and report poor quality of life [7, 10]. While 
the relationship between symptom severity and quality of life is well established, few studies indicated that the status of disability and its relationships with symptoms severity and quality of life. Therefore, the aim of this study was to describe the prevalence of disability, levels of symptom severity, and quality of life in a cohort of Chinese people living with SMI.

\section{Methods}

\section{Participants}

The SMI patients who met the following inclusion criteria were invited to participate in the study on their planned discharge: (1) 18 years old or more, (2) suffering from schizophrenia and related disorders, severe depression and bipolar affective disorder using the consensus diagnosis according to DSM-V, of American Psychiatric Association 2013, (3) having a long history of previous hospitalizations or outpatient treatment (at least 3 months), and (4) voluntary consent. Patients excluded from the cohort were under 18 years old, suffering from personality disorders, organic brain disease or learning disability, and refusing to give consent.

\section{Assessments}

This study used the World Health Organization (WHO)Disability Assessment Schedule-II (WHO DAS-II) [12], the Positive and Negative Syndrome Scale (PANSS) [13], and the WHO-Quality of Life-BREF version (WHOQOLBREF) [14] to assess disability status, symptom severity, and quality of life. A demographic sheet was used to collect patients' socio-demographic and clinical information.

\section{Data Collection and Analysis}

Data were collected from May 2014 to July 2014. Trained research nurses collected the data in face-to-face interviews 1 week before hospital discharge. Patients' voluntary participation was always observed. All data were coded and no personal identifiers appeared in the computerized data set. Data were analyzed using SPSS for Windows, version 21.0 by descriptive statistics, comparison and regression analysis. The level of significance was set at $P<0.05$.

\section{Ethical Issues}

This study was approved by the Third Affiliated Hospital of Guangzhou Medical University's Ethics Committee (No. 20140301). Patients were informed of the study aims, were given a Patient Information Leaflet and signed a research consent form.

\section{Results}

A total of 158 subjects participated in this study. These subjects were mainly suffering from schizophrenia or related disorders $(\mathrm{n}=73,46.2 \%)$. Their ages ranged from 18 to 65 , with a mean age of $33.10(\mathrm{SD}=10.62)$. Age of mental illness onset was $26.31(\mathrm{SD}=8.71)$. Table 1 shows detailed patient demographic characteristics.

Means of assessment outcomes is listed in Table 2. Regarding disability by WHO DAS-II, higher scores indicate higher levels of disability. Subjects perceived higher levels of disability in the aspects of social participation, getting along with others and dealing with life activities. For QOL by WHOQOL-BREF, patients had better QOL in the environmental domain than in the psychological and social domains. Concerning symptom severity by PANSS, higher scores indicate greater symptom severity. Participants had more severe general psychopathology symptoms than positive and negative symptoms.

Pearson correlation analyses were used to examine correlations of disability with symptom severity and QOL (Table 3). Overall, total disability scores were significantly correlated with patients' QOL and symptom severity. From Table 4, the prevalence of self-reported disability was as high as $69.6 \%(n=110)$. Those patients reporting a disability perceived higher levels of symptom severity and poorer QOL.

In the logistic regression analysis, there were two significant demographic predictors identified for disability status (Table 5). Older age was a significant risk factor for disability (OR 1.425, $95 \%$ CI 1.016-1.999). Shorter duration of current hospitalization was a significant protecting factor for disability (OR 0.704, $95 \%$ CI 0.508-0.974).

\section{Discussion}

This study found the prevalence of disability among Chinese people living with SMI was high. Common disability occurring due to SMI included participation in society, getting along with others and dealing with life activities. These findings were consistent with previous research [7, 8]. Risk factors related to disability for Chinese people living with SMI included older age and longer duration of hospitalization. As we all know, older people are more likely to be vulnerable when they are disabled. Longer duration of hospitalization may be related to more severe 
Table 1 Demographic and clinical characteristics of patients $(\mathrm{N}=158)$

\begin{tabular}{ll}
\hline Characteristics & $\mathrm{n}(\%)$ \\
\hline Psychiatric diagnosis & $73(46.2)$ \\
Schizophrenia and related disorder & $48(30.4)$ \\
Severe depression & $25(15.8)$ \\
Bipolar affective disorder & $12(7.6)$ \\
Others (e.g. missing) & \\
Gender & $89(56.3)$ \\
Male & $69(43.6)$ \\
Female & Mean (SD) $=33.10(10.62)$ (range 18-65) \\
Age (years) & $53(33.5)$ \\
Education level & $59(37.3)$ \\
Primary school or below & $46(29.2)$ \\
Secondary school & \\
College/university education & $20(12.7)$ \\
Residential status & $132(83.5)$ \\
Living alone & $6(3.8)$ \\
Living with family/relatives/friends & Mean (SD) $=26.31(8.71)$ (range 12-53) \\
Others (reluctant to tell or missing) & Mean (SD) = 8.27 (7.66) (range 0.3-36) \\
Age at diagnosis (years) & Mean (SD) = 56.80 (47.96) (range 12-380) \\
Duration of mental illness (years) & \\
Duration of current hospitalization (days) & \\
\hline
\end{tabular}

Table 2 Patients' measured mean scores of health outcomes

\begin{tabular}{lc}
\hline Instruments and score range & Mean (SD) \\
\hline WHO-DAS II-total score (0-48) & $7.89(7.70)$ \\
Cognition (0-8) & $1.59(1.39)$ \\
Mobility (0-8) & $1.22(0.89)$ \\
Self-care (0-8) & $1.60(0.89)$ \\
Getting along (0-8) & $1.66(1.17)$ \\
Life activities (0-8) & $1.61(1.51)$ \\
Participation in society (0-8) & $1.85(1.82)$ \\
WHOQOL-BREF-total score (24-120) & $76.30(12.33)$ \\
Physical health (7-35) & $22.15(3.65)$ \\
Psychological health (6-30) & $18.42(3.47)$ \\
Social relationship (3-15) & $9.90(2.34)$ \\
Environment (8-40) & $25.40(4.76)$ \\
PANSS-total score (0-180) & $52.60(26.61)$ \\
Positive symptoms (0-42) & $12.49(7.02)$ \\
Negative symptoms (0-42) & $12.73(7.67)$ \\
General psychopathology symptoms (0-96) & $27.43(13.93)$ \\
\hline
\end{tabular}

PANSS Positive and Negative Syndrome Scale, WHO-DAS II World Health Organization Disability Assessment Schedule II, WHOQOL$B R E F$ World Health Organization Quality of Life-Brief

disease conditions, so these people were more likely to be disabled.

SMI has a dramatic impact on various areas of life. Disability arising out of SMI disrupts the day-to-day life
Table 3 Correlations among patient's disability score with symptom severity and QOL

\begin{tabular}{ll}
\hline Pearson correlation coefficients & WHODAS II-total \\
\hline WHOQOL-BREF-total & $-0.433^{* *}$ \\
Physical health & $-0.415^{* *}$ \\
Psychological health & $-0.410^{* *}$ \\
Social relationship & $-0.356^{* *}$ \\
Environment & $-0.355^{* *}$ \\
PANSS-total & $0.616^{* *}$ \\
Positive symptoms & $0.396^{* *}$ \\
Negative symptoms & $0.511^{* *}$ \\
General psychopathology symptoms & $0.648^{* *}$ \\
\hline
\end{tabular}

** $P<0.001$

functioning of a person, self-care, interpersonal relationships, and social involvement [7]. This study indicated that the disability was more severe, it was significantly correlated with greater symptom severity and poorer quality of life. As disruption in social functioning also adds to the disability burden, people living with SMI found that it was extremely difficult to initiate and pursue any form of social activity [7]. Therefore, future psychiatric rehabilitation interventions should be developed to reduce the impact of SMI on people's social functioning.

The current study had two limitations. Although this study adopted a cohort study sampling, it took a cross- 
Table 4 Comparison of symptom severity and quality of life between nondisabled and disable patients by WHO-DAS II

\begin{tabular}{lllrr}
\hline Variables and domains & \multicolumn{2}{l}{ Mean $(\mathrm{SD})$} & \multirow{2}{*}{$t$} & \multirow{2}{*}{$P$} \\
\cline { 2 - 3 } & $\begin{array}{l}\text { No disability } \\
(\mathrm{n}=48)\end{array}$ & $\begin{array}{l}\text { Disability } \\
(\mathrm{n}=110)\end{array}$ & & \\
\hline WHOQOL-BREF-total & $81.72(11.33)$ & $73.39(11.91)$ & 3.80 & $<0.001$ \\
Physical health & $23.93(3.55)$ & $21.31(3.40)$ & 4.33 & $<0.001$ \\
Psychological health & $19.89(3.15)$ & $17.75(3.41)$ & 3.63 & $<0.001$ \\
Social relationship & $10.85(1.95)$ & $9.43(2.38)$ & 3.51 & 0.001 \\
Environment & $27.80(4.95)$ & $24.33(4.29)$ & 4.35 & $<0.001$ \\
PANSS-total & $41.63(23.05)$ & $58.28(26.66)$ & -3.59 & $<0.001$ \\
Positive symptoms & $10.76(7.41)$ & $13.29(6.73)$ & -2.06 & 0.041 \\
Negative symptoms & $9.65(6.63)$ & $14.06(7.74)$ & -3.36 & 0.001 \\
General psychopathology symptoms & $21.61(10.15)$ & $30.43(14.69)$ & -3.68 & $<0.001$ \\
\hline
\end{tabular}

PANSS Positive and Negative Syndrome Scale, WHO-DAS II World Health Organization Disability Assessment Schedule II, WHOQOL-BREF World Health Organization Quality of Life-Brief version

\begin{tabular}{llllll}
\hline Variables & B & SE & $P$ & OR & $95 \%$ CI \\
\hline Age & 0.354 & 0.173 & 0.040 & 1.425 & $1.016-1.999$ \\
Duration of current hospitalization & -0.352 & 0.166 & 0.034 & 0.704 & $0.508-0.974$ \\
\hline
\end{tabular}

Table 5 Significant factors related to patients' disability by logistic regression
Acknowledgments This study was funded by Guangzhou Medical University Fund for Overseas Returners (No. 2013C57).

\section{Compliance with Ethical Standards}

Conflicts of interest The authors have no funding or conflicts of interest to disclose.

\section{References}

1. Charlwood P, Mason A, Goldacre M, Cleary R, Wilkinson E, editors. Health outcome indicators: severe mental illness. Report of a working group to the Department of Health. Oxford: National Centre for Health Outcomes Development; 1999.

2. World Health Organization. Addressing global mental health challenges. Geneva: WHO Press; 2009.

3. China-CDC, National Center for Mental Health in 2009. http:// www.ncmh-ccdc.com/. Accessed on 28 June 2015. (in Chinese).

4. Adelufosi AO, Ogunwale A, Abayomi O, Mosanya J. Socio-demographic and clinical correlates of subjective quality of life among Nigerian outpatients with schizophrenia. Psychiatry Res. 2013;209(3):320-5.

5. Xiang YT, Hou YZ, Yan F, et al. Quality of life in communitydwelling patients with schizophrenia in China. J Nerv Ment Dis. 2012;200:584-7.

6. Thirthalli J, Venkatesh BK, Naveen MN, et al. Do antipsychotics Do antipsychotics limit disability in schizophrenia? A naturalistic comparative study in the community. Indian $\mathrm{J}$ Psychiatry. 2010;52(1):37-41.

7. Raj S. Living with a disability: a perspective on disability in people living with schizophrenia. Int J Psychosoc Rehabil. 2013;18(1):115-23.

8. Velthorst E, Nieman DH, Linszen D, et al. Disability in people clinically at high risk of psychosis. $\mathrm{Br} \mathrm{J}$ Psychiatry. 2010;197:278-84. 
9. Kao YC, Liu YP, Chou MK, Cheng TH. Subjective quality of life in patients with chronic schizophrenia: relationships between psychosocial and clinical characteristics. Compr Psychiatry. 2011;52:171-80.

10. Zeng Y, Zhou Y, Lin J, Zhou Y, Yu J. Generic and diseasespecific quality of life and its predictors among Chinese inpatients with schizophrenia. Psychiatry Res. 2015;228(3):724-8.

11. Zahid MA, Ohaeri JU, Elshazly AS, et al. Correlates of quality of life in an Arab schizophrenia sample. Soc Psychiatry Psychiatr Epidemiol. 2010;45(9):875-87.
12. World Health Organization. Disability assessment schedule II (WHO-DAS II). Geneva. http://www.who.int/icidh/whodas/who dasversions/12int.pdf. Accessed 15 May 2015.

13. Kay SR, Fiszbein A, Opler LA. The Positive and Negative Syndrome Scale for schizophrenia. Schizophr Bull. 1987;13:261-76.

14. WHOQOL Group. Development of the World Health Organization WHOQOL-BREF quality of life assessment. Psychol Med. 1998;28:551-8. 